\title{
Particle Physics Outreach: What we are doing and what we could be doing
}

\author{
Steven Goldfarb ${ }^{1}$ \\ University of Melbourne \\ Parkville VIC 3010, Australia \\ E-mail: steven.goldfarbecern.ch
}

This note reports on current education and outreach activities coordinated and supported by the LHC collaborations. These include a growing variety of efforts designed to inform the public, educate students, and communicate our progress to policy makers around the world. It also emphasises the importance of these efforts, given the recent rise of anti-science sentiment in influential political arenas, as well as the effect of social media as a growing platform for disinformation. The note further describes support provided by CERN and IPPOG to create highquality, coherent content backing these efforts, as well as a platform for the effective development and sharing of projects and ideas. Finally, the note underlines progress being made by the experiments to recognise and support outreach as an important responsibility for all collaboration members, and by some institutes to encourage outreach as a requirement of scientific research. It concludes by pointing out certain areas where more work is needed.

Sixth Annual Conference on Large Hadron Collider Physics (LHCP2018)

4-9 June 2018

Bologna, Italy

\section{${ }^{1}$ Speaker}




\section{Introduction}

\subsection{Why outreach}

We all agree it is an essential responsibility for scientific collaborations to present the results of their research to their peers for review, validation and the advancement of the body knowledge. It is equally important to present these results to scientists in other fields, to the public who have provided resources for the research, and to the funding agencies who have identified the field as a high priority, including it in their budgets. These efforts, however, can be challenging when the complexity of the field renders it difficult for non-specialists to comprehend our advancements or to understand their impact.

Efforts in particle physics outreach attempt to bridge this communication gap by developing content, channels and methods that allow us to clarify key concepts and to effectively summarise results in an interesting and comprehensible manner. Equally important are efforts to stimulate interest in the field, in order to reach new audiences who might potentially contribute to future research, either through direct participation as students or indirectly by helping to define national priorities as voting citizens.

\subsection{Further motivation}

The development of the world-wide web [1] at CERN [2] and its subsequent fast propagation around the globe has paved the way for cheap, accessible communication among most of the planet's population. In addition, the development of relatively inexpensive powerful computing and communication tools have made it possible for these users to access more information than we ever could have imagined a few decades ago.

Unfortunately, along with the power to inform with evidence-based information, the worldwide web brings the power to confuse with lies and propaganda. The authors of these acts of deception are not limited to internet trolls and others looking to entertain themselves at the expense of the innocent, but also include those seeking to make political or financial gain by casting doubt on facts and those who report them (Figure 1). This is a danger for science and for

society at large, and is something our field cannot Figure 1. One of our challenges.

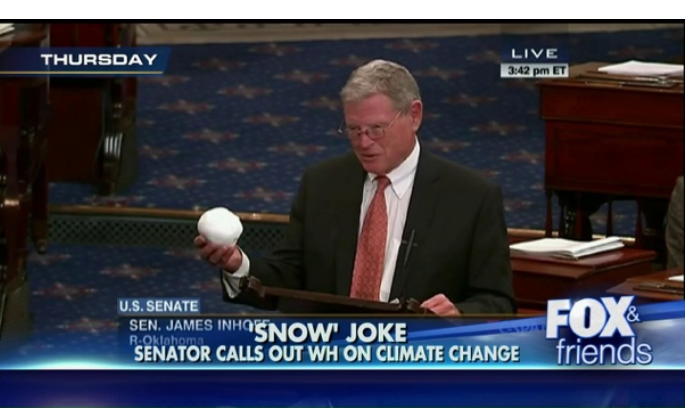
ignore.

There has always been a need for those of us involved in particle physics outreach to include an introductory component on scientific process in our educational programmes. More recently, however, many of us find the need to include discussion on philosophical and ethical issues, such as the existence of truth and the importance of validation and peer review. Such issues affect aspects of life beyond science and thus need to be handled in a sensitive manner. However, as experts in the pursuit of truth, we should not shirk the responsibility to remind humanity of its existence. It is a moral and necessary obligation, which only adds to the urgency of our work. 


\subsection{This document}

This document aims to highlight efforts currently being made by the particle physics community to develop, maintain, and improve outreach programmes around the world. It begins with an overview of efforts by the LHC collaborations, focusing on improvements to support mechanisms and process, rather than on specific projects, as these can change rapidly with time. It also presents a brief summary of the group at CERN supporting education and outreach for the laboratory, as well as the experiments, then describes the structure and activities of IPPOG [3], an umbrella particle physics outreach network that includes CERN and the experiments among its members. The document concludes by examining the progress our community has made in its recognition of outreach as an important component of our research, then points out weaknesses that still need to be addressed.

\section{The LHC Collaborations}

\subsection{Organisation}

The four major LHC experiments, ALICE [4], ATLAS [5], CMS [6], and LHCb [7], each host mature, well-organised education, outreach, and communication programmes. Modest core teams, typically including a few physicists and communication experts, develop and maintain platforms and content in support of their collaboration's external outreach efforts. These teams are usually run by one or two coordinators either nominated by their management or elected by their collaboration board. Other scientists or communication experts might lead specific projects, such as writing, multimedia, web development, social media, open data tool development, merchandising, exhibits, etc. The size of the effort, of course, depends on the investment of the collaboration, and varies between the experiments.

It should be emphasised that these teams do not in any way represent the full extent of outreach being undertaken by the experiments. Many of their member institutions and/or countries host important local programmes. These can include participation in local science festivals, public talks, exhibits, school programmes, etc. The core teams help to support these events by providing material, such as brochures, stickers, games, books, etc. and by using communication platforms, such as social media, for publicity.

Outreach core team members might receive compensation for their efforts in a variety of ways. Physicists, for example, could earn service credit for their home institutions in a similar manner to shift-taking or other operation tasks. Communication expertise provided by a member institution for specific services, such as writing or web development, could be compensated as part of a service contract between the experiment and that institution. Short-term (2-3 month) internships of communication students and longer-term (2-3 year) fellowships are also possible, but funding is limited.

\subsection{Target Audiences, Goals, Messages}

The primary goal of the experiments' outreach efforts are to communicate the scientific goals and accomplishments of their collaborations. To a large extent, this goal depends upon and overlaps with the need to educate the public on particle physics and science, in general, and on convincing various audiences on the value our field brings to society. Without establishing an understanding of the large philosophical questions being addressed by our field and how seeking 
solutions to these problems advances humanity, justification of support can be very difficult. The experiments develop this foundation by addressing a range of audiences with clear, targeted messages.

\subsubsection{Students}

Perhaps the most important and challenging of these audiences (arguably the most fun) are children. In this case, slight differences in age can make significant differences in the type and complexity of the messages to convey, as well as to the methodology of delivery. For many years, the primary age group for education and outreach efforts has been secondary school, typically between ages 14 and 18 .

In this age range, it is thought that students have the necessary mathematical and scientific

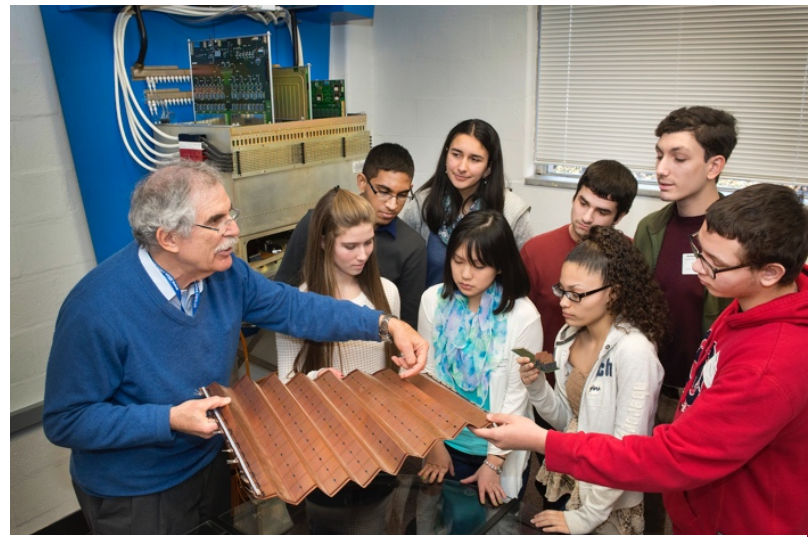

Figure 2. Outreach to secondary school students. background to be able to understand the basic concepts of particle physics. These include topics, such as vector addition, conservation of charge, momentum and energy, mass-energy equivalence, etc. In this case the goal is to build upon these basic skills by showing how they can apply to the microscopic world of particle physics. Lessons introduce a few basic concepts from both the theoretical and experimental sides of our field (Figure 2), in order to give the students a taste of what we do. The key message here is that particle physics is fun and accessible, and a field they might consider pursuing in the future.

More recently, important efforts have been made to reach younger audiences, including those in the earliest stages of secondary school, commonly referred to as middle school (ages 1113), and those in primary school (ages 10 and under). As expected, these age groups present a much broader range of skills, abilities, and attention spans, so methods and messages vary across the spectrum.

In some cases activities designed for the secondary school students are re-designed to work for audiences with a less advanced mathematical or scientific background. Calculations can be replaced with simple questions that are more qualitative than quantitative, in nature. Often, however, a completely new approach is more appropriate. For example, students can be encouraged to express their understanding of the material by playing games, creating art, or storytelling, rather than through traditional charts, plots, and graphs.

Addressing these younger age ranges forces the primary message to shift from content to process. In this case, the educators focus on describing the general usage of evidence and measurement to understand nature and to uncover the underlying rules. This allows the students to recognise patterns in everyday life, through analogy, and helps teach them the valuable lessons of basing decisions on fact.

Interestingly, this shift of emphasis toward explaining scientific process and methodology to younger audiences has propagated forward to educational programmes with older students, and up the age ladder to programs targeting the general public. It is arguably one of the most important 
messages to be shared, especially in a time of extensive overt misinformation campaigns involving science, including topics like vaccine warnings, fake medicine, and even a flat earth. The goal here is that all audiences gain an appreciation of how science helps us to differentiate fact from fiction, thus allowing humankind to advance.

\subsubsection{Policy Makers and the Public}

Another key audience often identified independently by the experiments is that of policy makers or decision makers. These are defined as leaders in government, industry, and science

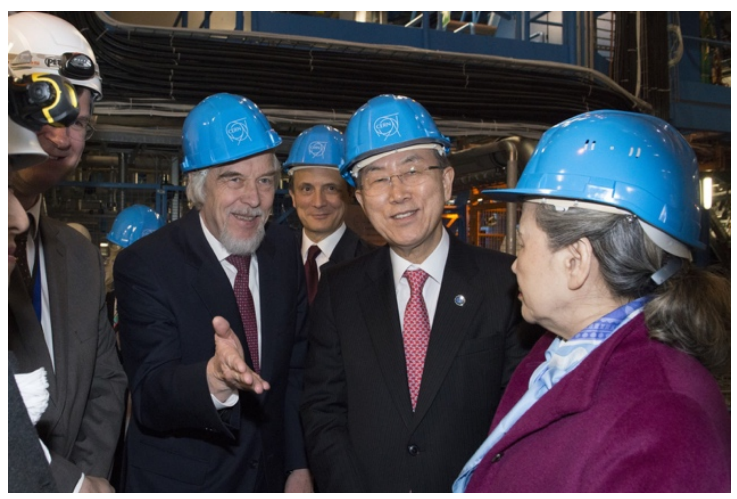

Figure 3. Targeting policymakers. policy whose decisions can have an immediate and/or long-term impact on the field and beyond (Figure 3). The primary goal of reaching these audiences is to secure support for current and future scientific programmes and, perhaps, to remind them how to make important decisions when running their businesses or governing our countries. In this case, most messaging focuses on the important impact our research has on society, including long-term security and economic strength, in addition to the noble vision of improving human understanding of our universe.

These messages are essentially equivalent to those targeting the public, in general. What can vary between the two groups is the emphasis on immediate return versus long-term investment. The public tend to be more open to broad discourse on humanity's quest for knowledge, while policy makers often require sharp talking points on specific gains to be made in their industry or region: employment, education, industrial spin-offs, etc. Regardless, it is imperative to deliver the same messages to the public, as they ultimately select and influence the policy makers.

\subsubsection{Other Scientists}

It is important not to neglect scientists, both in and out of the field of particle physics, as a key target audience. Specialised nomenclature, acronyms, and scientific methodology can render technical documents and scientific publications difficult to understand even for those in the same field. Well-written summary articles, images, videos, and animations can help to clarify concepts and also to provide tools to be used by the scientists for their own outreach efforts. In this case, the key messages not only include examples and analogies for them to share, but also to help them to gain a stronger overall understanding of their own field.

\subsection{Platforms, Content, Activities}

\subsubsection{Public Web Sites}

The primary online platforms for the LHC experiments are their public web sites. These sites not only provide the public with descriptions of the experiments, as well as the latest news of their plans and accomplishments, but also support the outreach efforts of the collaborations by hosting educational tools and content. The web sites are also used to publicise activities of the member institutes and countries, and to provide access and visibility to social media and other forms of communication. 
The four major LHC experiments each have attractive, well-designed, content-rich public web sites [4-7]. CMS and ATLAS have moved from standard html-based sites to content management systems, in order to facilitate growing communication needs, improve crossreferencing of related content, and allow for web-based content development and maintenance. The ALICE experiment is in the process of migrating in the same direction; LHCb currently has no such plan. The content management system of choice is Drupal ${ }^{2}[8]$, as it is recommended and supported by CERN.

\subsubsection{Social Media}

Over the past 5-6 years, social media has rapidly grown as the dominant force in online communication, and the LHC experiments have been adapting their content and communication strategies to incorporate it as a serious platform. Early on, the primary media in use by the experiments were Facebook, Twitter, Google+, YouTube, and Flickr. More recently, Instagram has come into play for hosting images and video, and several experiments have dropped usage of Google + , as activity on that platform has diminished.

Facebook and Google+ usage for many years focused on grabbing the reader's attention with titles, high quality imagery and ledes (short summary introductions) or excerpts from content published on the public web site. Videos and gif files have been added, as the functionality was made available. Twitter is used in the same manner, albeit without the ledes, due to lack of space. It is also used to share relevant content from other web sites, including articles written by collaboration members, announcements for events, etc.

The original strategy was to drive the reader to the public web site with the primary story and hope they would stay to read other content about the experiment. Web site statistics clearly indicate a strong correlation between social media posts and increased viewing of the web sites. But, while the posts were driving viewers to the public web site, they were even more effective at increasing membership on the social media sites, themselves. The growing number of followers and increased activity on the social media sites led the experiment communication teams to realise that social media is an important platform on its own for delivering content. With improvements in the delivery mechanisms, such as Facebook article editing and publishing, more tools are available for reaching audiences that prefer not to leave their favourite social media platform.

Another key factor in the development of communication strategy and content delivery techniques has been the tremendous growth in the usage of smart phones for content access. This has affected the design of the public web sites, in that their pages need to be effectively viewed on smaller screens. It has also affected the development of multimedia content for both web sites and social media. The duration and shape of videos need to cater to expected smart phone usage, which typically implies concise messages and appropriate (typically square or vertical) formats. Live video productions have moved from Google Hangouts (a Google+ product) to Facebook Live, and Instagram is now creating a market for video, called IGTV.

In summary, social media has become a key player for delivery of online content, and has helped the communication teams to reach new audiences. It is a quickly evolving field, which demands flexibility in both strategy and content delivery techniques. But, it has tremendous potential, when used effectively.

${ }^{2}$ All trademarks in this article are acknowledged as such. 


\subsubsection{Exhibits and Events}

The LHC experiments all have a wide variety of programs designed to inform and satisfy the curiosity of the public, as well as the inquisitive minds of young students. At CERN in 2017, roughly 135,000 people came to visit CERN, to see the facilities, and to better understand what the LHC experiments are working to achieve. The vast majority of these visitors were students, mainly in secondary education or university.

Exhibits or Visitor Centres have been set up at the location of each of the LHC experiments. Visitors on guided tours receive information about the experiments, their physics goals, the functioning of large international collaborations, the technical challenges of the detectors, and how they handle the large quantity of data they record. The guides for these visits range from professionally trained CERN guides to members of the collaboration, requested for the visit because of their language, nationality, institutional affiliation, or simply because they enjoy talking about their work. When the LHC is accessible, during year-end (a few months) or long (a few years) shut-downs, guides are able to bring their visitors underground, a very popular activity.

To complement these activities, and to reach remote groups who are unable to make the visit to CERN, the experiments have set up Virtual Visit facilities [9]. Guides at the control rooms or underground, talk to remote audiences via videoconference and use specially set up cameras to give remote tours of the location. These visits are often recorded for the benefit of family, friends and

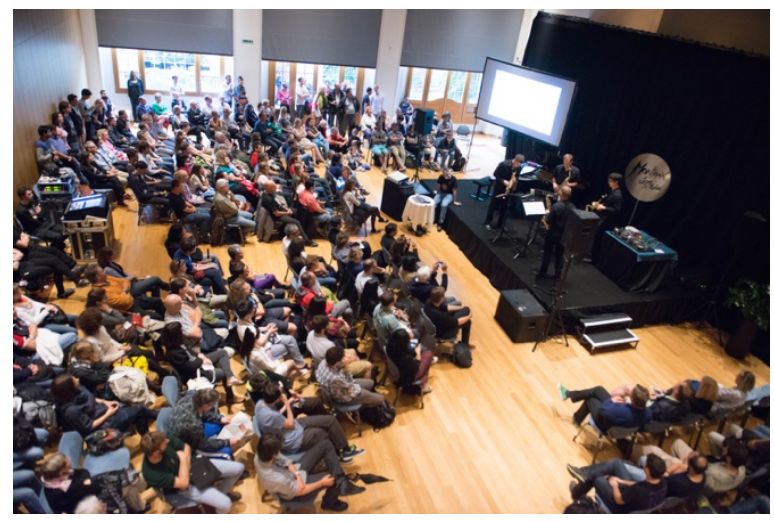

Figure 4. Reaching a new audience at the Montreux Jazz Festival. schoolmates. Virtual Visits are an excellent, light-weight method for reaching into classrooms and sparking the interest of students by taking time to answer their questions about the experiments and the field of particle physics.

Beyond visits hosted at CERN, the LHC experiments participate in public events hosted at CERN on special occasions, such as Open Days, or hosted in remote locations, as a part of another event, such as a science festival, art or music festival (Figure 4), or physics conference. The outreach teams help to organise the activities, prepare material, and coordinate efforts by their collaboration members. Public outreach events held in conjunction with major particle physics conferences, such as ICHEP [10], LHCP [11] or CHEP [12], have become more common in recent years, as they engage local audiences and give the participants a chance to hone their communication skills.

\subsubsection{Informal Education}

Outreach teams of the LHC experiments have all engaged in the development of materials and programmes in support of informal education, for all ages, from primary school, through university. The list is extensive and we will not try to include them all here. A few notable highlights include CMS Playing with Protons [13], the ATLAS Colouring Book [14], ALICE Papercraft [15] and Open Data Portals [16] for all the experiments, designed to give access to data and tools for students to learn and perform physics analysis. Many more programmes and much 
more material can be found on the experiments' public web sites. The LHC experiments are all also actively involved in the development and hosting of Particle Physics Masterclasses [17], which we will describe in the IPPOG section below.

The educational initiatives of the outreach teams are meant to complement the scientific education programmes of formal schooling and to provide opportunities that might not be available in all countries or to all members of a society or socio-economic class. Partnerships are often sought between formal educators and researchers to implement the programs in the most effective manner, with each providing their own expertise. Teachers appreciate the detailed information the researchers can bring to respond to student questions, while researchers benefit from the methodologies and pedagogical expertise provided by the educators.

\section{Support from CERN and IPPOG}

\subsection{CERN}

CERN is the host laboratory for the LHC experiments and is thus responsible for providing infrastructure and facilities required for the functioning of the detectors and the large international collaborations that build and run them. In a similar manner, the CERN Education, Communication and Outreach (ECO) team provides support for the much smaller LHC collaboration teams, in addition to promoting the goals of the laboratory, itself. As one can guess, these goals are similar and overlapping, since the success of the experiments and the laboratory are deeply interwoven. However, the teams operate independently and benefit tremendously from their own originality, skills, and imagination.

We will not attempt to summarise the organisation and functioning of the CERN ECO group in this document, for lack of space, but will refer to their web site [18], which describes the mandate, activities, strategy, and structure of the group. Interactions and coordination between CERN ECO and the experiments occur weekly, during an Editorial meeting, every two months, during the LHC Outreach Group (LOG) meeting, and in a variety of ad-hoc meetings set up to address specific communication or outreach activities.

Activities that require significant resources, such as the production of videos, animations, graphic design, and photography are provided by the CERN ECO team for the experiments, when they do not have the means to produce them on their own or when there is a common need. CERN also hosts infrastructure for the experiments' internal and external web pages and dedicated database services for the storage of written material, images, video and animations.

LHC collaboration members can participate in CERN training to learn communication skills or become guides for the CERN Visit facilities. Both CERN ECO and the experiment teams are pro-active in seeking partnerships with external education and communication specialists, inviting them to visit CERN, and often organising workshops with open participation. Unlike physics analysis teams in competing experiments, the LHC outreach teams share a significant amount of knowledge and ideas, as they benefit from each other's success.

\subsection{IPPOG}

The International Particle Physics Outreach Group (IPPOG) is a global network of scientists, science educators, and communication specialists actively engaged in particle physics outreach. It began as the European Particle Physics Outreach Group (EPPOG) in 1997, but has grown to 
become a global scientific collaboration, the members of which include 28 countries, 6 particle physics experiments, and 2 laboratories, DESY and CERN. Twice a year, representatives from these members join other science education and communication specialists to report, discuss, and develop best practices in particle physics outreach.

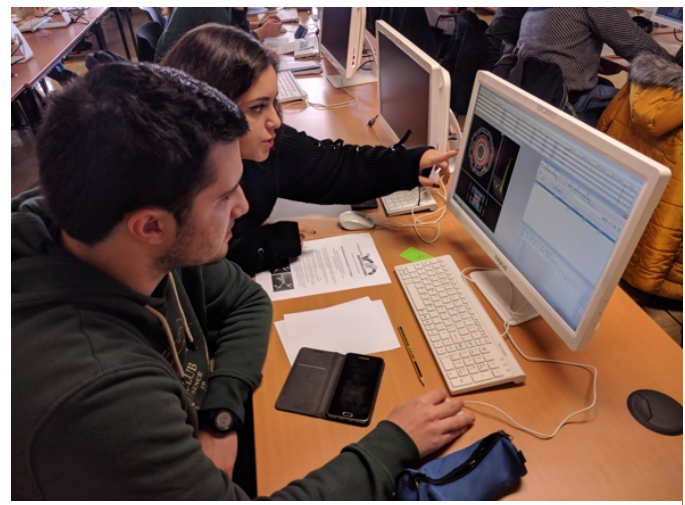

Figure 5. Students in an IPPOG Physics Masterclass.

One of the most important initiatives to come from IPPOG is the International Physics Masterclass programme, which allows thousands of students around the world to learn about and analyse real data from current, cutting-edge particle physics experiments. In Spring 2018, IPPOG coordinated 307 Masterclasses in 52 different countries, reaching more than 14,000 students around the globe (Figure 5). These students meet physicists from the experiments, learn how to carry out research in an international collaboration, and become scientists for a day, analysing real data and comparing their results with their colleague-classmates.

Other major activities developed and coordinated by IPPOG members include a Global Cosmics portal [19], hosting links to worldwide projects designed to give students the opportunity to explore cosmic particles, an Exhibits working group, which seeks to create and share plans for the development of effective particle physics exhibits and components for museums and other public spaces, and a working group exploring methods for explaining physics hot topics to a lay audience. Meetings include presentations by these working groups, as well as panel discussions on topics, such as Physics \& Beauty and the Outreach of Applications of Particle Physics for Society.

Members of the laboratory and experiment outreach teams have a chance during these meetings to develop and share ideas with national representatives. The networks created by IPPOG allow for researchers and outreach content developers to have more direct links with teachers and students around the world, so they can work together to create programmes optimised for particular regions, languages, and cultures.

\section{Conclusion}

\subsection{Recent Progress}

The LHC experiments have made important progress over the past few decades in the advancement of particle physics outreach. Public communication and education are no longer considered as "extra" activities by a few scientists speaking to classrooms, hosting the media or giving public talks, but are considered essential components of the field. Small but professional teams on the experiments partner with experts in education and communication to develop mature strategies, content, and platforms targeting specific audiences around the world.

CERN and IPPOG work together with the experiments to develop content, coordinate programmes, and share best practices. All teams have worked hard to reach remote audiences via traveling exhibits and classroom visits, and using modern communication tools and platforms, including social media and live streaming. Content development and educational methodologies 
continue to evolve to promote the clear effective communication of concepts, while simultaneously stimulating the imagination and appreciation of the public.

Although success of the programmes is difficult to quantify, it is evident in the large audiences that witnessed the turn on of the LHC and the discovery of the Higgs boson. Audience size and engagement continue to grow on the online platforms and, in many countries around the world, student enrolment in physics is on the rise. Recognition within our own field of the importance of education and outreach is evident in the growing number of major particle physics conferences that host concurrent public events and/or dedicated plenary talks and/or sessions. It should also be noted that a number of institutes, countries, and funding agencies now request a component of outreach be included in project grant proposals, a major step forward.

\subsection{What Remains}

Despite the progress, there remain important steps to be taken to foster recognition of the skills developed by participants of the education and outreach programmes. Anyone involved in public outreach realises the challenges and value of the discipline. In addition to developing education and communication skills, scientists are forced to rethink their own understanding of the field. In order to effectively communicate the goals and accomplishments of particle physics, one needs to understand clearly the implications of our work and the role it plays in society.

These skills are essential for any young physicist who seeks a career as university faculty or laboratory staff. It would thus seem natural for physics departments and hiring boards to seek applicants with experience in education and outreach, in support of their skills as researchers, but this is rarely the case. Most young scientists in graduate school or postdoctoral positions find themselves discouraged from "wasting time" on trivial activities, such as outreach, as this might take time away from their research efforts. That is a problem that needs to be solved.

Some institutes and countries are seeking to do just that. The US National Science Foundation employs two criteria [20] in the merit review of proposals: What is the intellectual merit of the proposed activity? What are the broader impacts of the proposed activity? A new Italian law of November 2016 requires all Italian research institutes to be evaluated not only for their research output but also for their Third Mission activities (to disseminate scientific culture and the organisation of internships for secondary school students) [21]. A recent job advertisement in the Czech Republic explicitly sought "Academic researcher at the Institute of Particle and Nuclear Physics oriented to experimental particle physics. Part of the job will be coordination of particle physics outreach." So, there is a hope that we are headed in the right direction. Let's encourage this progress, so that the next generations of researchers can have the opportunity to develop a broader and richer understanding of our field, and to be able to communicate that knowledge effectively to the world.

\section{References}

[1] Tim Berners-Lee, Information Management: A Proposal, CERN, May 1990.

[2] CERN is the European Organization for Nuclear Research, http://home.cern .

[3] IPPOG is the International Particle Physics Outreach Group, http://ippog.org .

[4] The ALICE Experiment at CERN, http://alice.web.cern.ch . 
[5] The ATLAS Experiment at CERN, http://atlas.cern .

[6] The CMS Experiment at CERN, http://cms.cern .

[7] The LHCb Experiment at CERN, http://lhcb-public.web.cern.ch .

[8] Drupal ${ }^{\mathrm{TM}}$ is a content management system deployed by CERN and used by the LHC experiments, http://drupal.org.

[9] S Goldfarb et al, Sharing scientific discovery globally: toward a CERN virtual visit service, 2017, J. Phys.: Conf. Ser. 898102005.

[10] ICHEP is the International Conference on High Energy Physics. The latest was held in Seoul, Korea in 2018, http://www.ichep2018.org .

[11] LHCP is the Conference on Large Hadron Collider Physics. The latest was held in Bologna, Italy in 2018, http://hcp2018.bo.infn.it .

[12] CHEP is the International Conference on Computing in High Energy Physics. The latest was held in Sofia, Bulgaria in 2018, http://chep2018.org .

[13] Playing with Protons is an education initiative led by CMS, https://playprotons.web.cern.ch.

[14] ATLAS Colouring Book, https://atlas.cern/colouring-book .

[15] ALICE Papercraft, http://alicematters.web.cern.ch/?q=PapercraftOpenDays2013 .

[16] CERN Open Data Portal, http://opendata.cern.ch .

[17] International Physics Masterclasses, http://physicsmasterclasses.org .

[18] The CERN ECO Group is described at https://communications.web.cern.ch .

[19] International Cosmic Day portal, http://globalcosmics.org .

[20] The US NSF Broader Impacts Review Criterion, https://www.nsf.gov/pubs/2007/nsf07046/nsf07046.jsp .

[21] The Third Mission statement for INFN, http://home.infn.it/en/9-comitati . 